\title{
Introducing a Calculus of Trees
}

\author{
S. Kasangian \\ S. Vigna \\ Dipartimento di Matematica, Università di Milano, \\ Via Saldini 50, I-20133 Milano MI, Italy
}

\section{Introduction}

Labelled trees are a basic tool in both theoretical and practical Computer Science. Different kinds of operations and constructions are used intuitively both in programming and in the study of sequential and parallel computations. For instance, a typical feature of Milner's CCS (see [Mil80]) is the manipulation of equations on infinite trees. Milner's RST are based on a set of unary operators on trees (left-prefix), one for each action, and on a commutative binary operation, and provide a descriptive approach to a "model" for CCS. Notice further that all the CCS-like approaches to parallel computations (for instance, Bergstra-Klop's Process Algebras [BK85]) lead to a similar intuition about trees, which has been also formalized in terms of category theory, for instance, by Winskel in [Win84] and, later, by Kasangian and Labella in [KL.90].

Very recently, in lectures held at the Mathematics Department and at the Computer Science Department of the University of Milano [Bén90], Jean Bénabou introduced the concept of motor, a simple algebraic structure which reflects very well the recursive nature of finite forests (and trees) and provides a uniform, clean and elegant treatment of them. The novelty of Bénabou's approach is that he makes extensive use of the initial motor (in a suitable category) with its powerful universal property, so that induction becomes a "built-in" feature: using very simple categorical tools Bénabou is able to characterize many attributes of forests (for instance, the number of nodes, of leaves etc.).

In the attempt of modelling labelled trees in Bénabou's framework, the authors were led to a natural generalization of the notion of motor, namely $\Sigma$-motors. In fact, not only RST's are $\Sigma$-motors, but in their very definition all the ingredients of a $\Sigma$-motor turned out to be actually present, and initial algebras are used from a long time, after all, in the study of trees.

The aim of the present paper is to start the foundation of a rigorous but manegeable calculus of finite labelled trees, combining the current intuitions of Computer Science and the power of the new algebraic structure. The notions we deal here with, like counting maps or tree products, are by no means new, but the point of view we introduce allows for a very simple yet powerful axiomatization of the concept of "finite tree" which is generalizable to an ambient topos or even to a distributive category (see [KV]), and is very well suited for actual computations (see for instance [DKV]). The account will be somewhat didactical due to the introductory nature of this work. 


\section{Fundamentals}

In order to develop our calculus, we start by defining $\Sigma$-motors.

\section{$2.1 \quad \Sigma$-motors}

Definition 2.1 Given an alphabet $\Sigma$, a $\Sigma$-motor is a tuple $\langle M, \oplus, 0, f\rangle$, where $\langle M, \oplus, 0\rangle$ is a monoid, and

$$
f: \Sigma \times M \longrightarrow M
$$

is a map.

The definition above generalizes Bénabou's notion of motor, that we recover when $\Sigma=\{*\}$, so that $f$ becomes an endomap of $M$. If $\alpha, \beta, \gamma, \ldots$ are elements of $\Sigma$, and $t$ is an element of $M$, we write $\alpha(x)$ (or even $\alpha x$ ) for $f(\alpha, x)$, according to the usage in $R$-module theory and $\Omega$-group theory. Indeed, $f$ should be viewed as an action of $\Sigma$ on $M$, like the external product in an $R$-module or in an $\Omega$-group. The axioms for a $\Sigma$-motor as given in Definition 2.1 are in fact a weakening of the axioms for a left $R$-module or a left $\Omega$-group. For the same reason we write $\alpha$ for $\alpha(0)$ (one-dimensional vectors can be identified with the elements of the ground ring). If needed, we will feel free to use

$$
\sum_{k} \sigma_{k} x_{k}
$$

to denote indexed sums, even if this kind of notation can be misleading, because it is used almost exclusively for abelian structures. The gain is of course a more compact display. Further, while dealing with actions $f$ which actually depend only on $M$, i.e., factor through the canonical projection $\pi_{M}: \Sigma \times M \longrightarrow M$, we will understand the latter, thus writing only the $\operatorname{map} M \rightarrow M$.

By cartesian closedness, an alternative definition could be given using an $\Sigma$-indexed family of endomaps of $M$. The resulting structure, which is basically a one-sorted algebra given by a monoid and by $|\Sigma|$ unary operators, is a slight weakening of Milner's RST's [Mil80]. The interplay between these two points of view turned out to be extremely productive. However, Definition 2.1 is suitable for wide generalizations [KV], while the one sketched above relies on being able to talk about "endomaps", which is impossible in general if the ambient is not cartesian closed.

Introducing a new $\Sigma$-motor, however, we will often prefer to state the definition in term of endofunctions, because the $R$-module-like notation permits a more readable description of $f$ (see examples in Sections 2.2 and 2.3).

As for the morphisms between $\Sigma$-motors, let us give the following

Definition 2.2 A morphism between two $\Sigma$-motors $\langle M, \oplus, 0, f\rangle$ and $\left\langle M^{\prime}, \oplus^{\prime}, 0^{\prime}, f^{\prime}\right\rangle$, is an arrow $A: M \longrightarrow M^{\prime}$ which is a monoid morphism and satisfies $A(\sigma t)=\sigma A(t)$, for all $t \in M$ and $\sigma \in \Sigma . \mathbb{M}_{\Sigma}$ will denote the initial object of $\Sigma$-Mot, the category of $\Sigma$-motors 1 .

\footnotetext{
${ }^{1}$ We write $\mathbb{M}$ for $\mathbb{M}_{\{*\}}$, the motor of unlabelled trees.
} 
In terms of the alternative definition (see above), the condition $A(\sigma t)=\sigma A(t)$ simply means that $A$ is equivariant with respect to all the endofunctions. This viewpoint is certainly intuitive, but Definition 2.2 generalizes to distributive categories (see [KV]), and, when expanded, forces pseudo-linearity conditions

$$
\begin{aligned}
A(0) & =0 \\
A(\sigma x) & =\sigma A(x) \\
A(x \oplus y) & =A(x) \oplus A(y)
\end{aligned}
$$

An arrow-only description of the condition $A(\sigma t)=\sigma A(t)$ is given by the equation $A \circ f=$ $f^{\prime} \circ\left(\mathbf{1}_{\Sigma} \times A\right)$.

Now we want to sketch some of the reasons justifying the use of $\Sigma$-motors to manage trees. Indeed, at first glance one could think that $\mathbb{M}_{\Sigma}$ is "too simple": after all, the initial object of Mon is the trivial monoid. But recall that $0 \in \mathbb{M}_{\Sigma}$, and that necessarily $\alpha(0) \neq 0$ for all $\alpha \in \Sigma$, by the initiality of $\mathbb{M}_{\Sigma}$. Further, still by initiality, we have that $\alpha(0) \oplus \beta(0)$ is a "new" element. These considerations show that $\mathbb{M}_{\Sigma}$ has infinite elements, but maybe they don't give an insight of its structure. So we start with some heuristic remarks, which are essentially an adaptation of Bénabou's account of unlabelled forests.

Let us consider an "object" $\mathbb{T}_{\Sigma}$ whose members are to be thought of as finite ordered trees labelled on their arcs. This object could have been defined in many different ways, and could also have been equipped with some operations. However, all we need to know is that we can generate a new tree either by joining the roots of a pair of trees (preserving the order), or by adding a new labelled arc at the top of a tree. This second operation (left-prefix) can of course be performed with each label, as in the following picture:

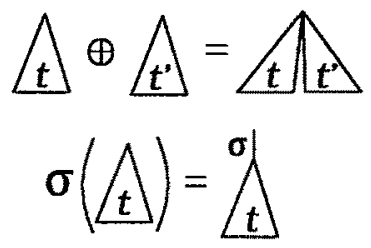

The "join" operation would be of course associative, and the "root-only" tree would play the rôle of the identity. So we would end up with a monoid. On the other hand, the "arc-creating" operations would define an action of $\Sigma$ on $\mathbb{T}_{\Sigma}$, so we would get a $\Sigma$-motor. Thus, we would have a unique arrow in $\Sigma$-Mot

$$
\mathrm{M}_{\Sigma} \stackrel{\text { Pict }}{\longrightarrow} \mathbb{T}_{\Sigma}
$$

since $\mathbb{M}_{\Sigma}$ is initial.

Let us now examine one basic property of labelled trees; we choose, for instance, the number of arcs, which is given by a map $n: \mathbb{T}_{\Sigma} \longrightarrow \mathbb{N}$. When we join two trees, we add the numbers of their arcs, and when we add a labelled arc at the top of a tree, the number of arcs increases by one. All this means that

$$
n: \mathbb{T}_{\Sigma} \longrightarrow\langle\mathbb{N},+, 0, s\rangle
$$


where $s$ is the successor function, is a $\Sigma$-motor map. But then, the unique map

$$
\nu: \mathbb{M}_{\Sigma} \longrightarrow\langle\mathbb{N},+, 0, s\rangle
$$

must factor through Pict, since by the initiality of $\mathbb{M}_{\Sigma}$ the diagram

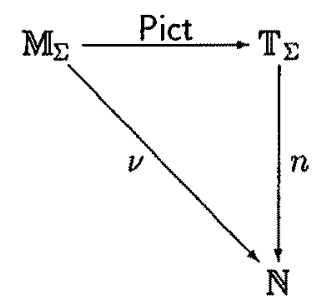

necessarily commutes. Hence the slogan: "count the $\operatorname{arcs}$ in $\mathbb{M}_{\Sigma}$ rather than in $\mathbb{T}_{\Sigma}$ ".

This process could be carried on again with a different $\Sigma$-motor, like $\langle\mathbb{N},+, 0,(-) \vee 1\rangle$, and we would obtain this way a map that counts the number of terminal arcs of a tree. Many other counting maps can be created this way (see the examples in Sections 2.2 and 2.3).

We hope we gave the reader an insight about the nature of $\mathbf{M}_{\Sigma}$. The operation $f$ creates new labelled arcs, $\oplus$ joins the roots and 0 is the root-only tree. Thus, $\alpha \oplus \beta$ and $\gamma(\alpha \oplus \beta)$ represent just:

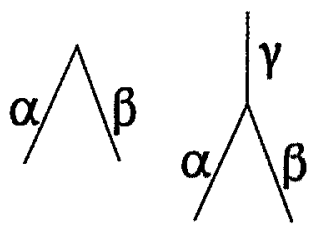

It's worth observing that we disregard the nature of $\mathbb{T}_{\Sigma}$. We only suggest that most (if not all) of the interesting properties of trees can be studied in $\mathbb{M}_{\Sigma}$, since most (if not all) of them should factor through Pict.

We note here that all we said above could be applied to the initial abelian $\Sigma$-motor, which can be thought of as the object of finite unordered labelled trees.

\subsection{Morphisms to $\mathbb{N}$}

In this section we will exhibit a few examples of counting maps which express basic properties of labelled trees just assigning the appropriate $\Sigma$-motor structure on the codomain, namely $\mathbb{N}$. The approach is the same as in the previous section: we look for properties which, in a sense, present a regular behaviour when you apply the $\Sigma$-motor operations.

The intuition behind the "initial object method" is that the unique map out of $\mathbf{M}_{\Sigma}$ "dismounts" a tree and "remounts" it using the endofunctions and the monoid operation defined on $\mathbb{N}$. Of course, the result is not any longer a tree, but rather a natural number. We will use $n$ to denote an arbitrary element of $\mathbb{N}$. 
Example 2.1 Let $T \subseteq \Sigma$. Call $\nu_{T}$ the unique morphism from $\mathbb{M}_{\Sigma}$ to $\left\langle\mathbb{N},+, 0, f^{\prime}\right\rangle$, where $\sigma n=n+1 \forall \sigma \in T, \sigma n=n \forall \sigma \notin T$.

Intuitively, $\nu_{T}$ counts the number of arcs with labels in $T$. Interesting special cases are of course $T=\Sigma$ (all arcs) and $T=\{\alpha\}$ (occurrences of a chosen label). For sake of simplicity, we write $\nu$ for $\nu_{\Sigma}$.

Example $2.2^{\prime}$ Let $T \subseteq \Sigma$. Call $\partial_{0}^{T}$ the unique morphism from $\mathbb{M}_{\Sigma}$ to $\left\langle\mathbb{N},+, 0, f^{\prime}\right\rangle$, where $\sigma n=1 \vee n \forall \sigma \in T, \sigma n=0 \vee n=n \forall \sigma \notin T$.

Again, $\partial_{0}^{T}$ counts the number of arcs with labels in $T$ which are not followed (at any depth) by other arcs with labels in $T$. Indeed, when two trees are joined, these numbers get added. When a new arc prefixes a tree $t$ with no arcs labelled in $T$, you count just one if the label of the arc was in $T . \partial_{0}^{\Sigma}\left(\partial_{0}\right)$ clearly counts all the terminal arcs (the leaves).

Example 2.3 Let $T \subseteq \Sigma$. Call $\partial_{1}^{T}$ the unique morphism from $\mathbb{M}_{\Sigma}$ to $\left\langle\mathbb{N},+, 0, f^{\prime}\right\rangle$, where $\sigma n=1 \forall \sigma \in T, \sigma n=0 \forall \sigma \notin T$.

It is obvious that $\partial_{1}^{T}$ counts the number of arcs immediately under the root (i.e., the branching degree) which have labels in $T$. Just like above, $\partial_{1}^{\Sigma}$ (briefly, $\partial_{1}$ ) counts them all.

Example 2.4 Let $T \subseteq \Sigma$. Call $\mu_{T}$ the unique morphism from $\mathbb{M}_{\Sigma}$ to $\left\langle\mathbb{N},+, 0, f^{\prime}\right\rangle$, where $\sigma n=1 \vee n \forall \sigma \in T, \sigma n=0 \forall \sigma \notin T$.

This map counts the number of arcs not followed by arcs in $T$ which are reachable passing only through arcs labelled in $T$, i.e., intuitively, in how many places you can arrive moving as far as possible only through arcs in $T$. As an example, think of the tree as the behavior of an agent. If $T$ represents the actions which don't allocate memory from the system, then $\mu_{T}$ represents the number of states one can reach without asking for new memory.

Example 2.5 Let $T \subseteq \Sigma$. Call $\rho_{T}$ the unique morphism from $\mathbb{M}_{\Sigma}$ to $\left\langle\mathbb{N},+, 0, f^{\prime}\right\rangle$, where $\sigma n=n+1 \forall \sigma \in T, \sigma n=0 \forall \sigma \notin T$.

This morphism counts the number of arcs occurring in paths labelled only in $T$. In other words, the number of places reachable moving only through $T$-actions.

If now we change also the monoid operation in $\mathbb{N}$ we can obtain some other interesting maps. We well be using mainly the operations $(-\vee-): \mathbb{N} \times \mathbb{N} \rightarrow \mathbb{N}$ (sup) and $(-\dot{\Lambda}-): \mathbb{N} \times \mathbb{N} \longrightarrow \mathbb{N}$, where $\dot{\Lambda}$ is defined as $a \wedge b$ if $a, b>0$, or $a \vee b$ if $a=0$ or $b=0$.

Example 2.6 Let $T \subseteq \Sigma$. Call $h_{T}$ the unique morphism from $\mathbb{M}_{\Sigma}$ to $\left\langle\mathbb{N}, \vee, 0, f^{\prime}\right\rangle$, where $\sigma n=n+1 \forall \sigma \in T, \sigma n=0 \forall \sigma \notin T$.

This map is very important, and counts the maximum length of a path starting from the root with labels only in T. In fact, if we join two trees $t, t^{\prime}$, we must take as $h_{T}$ of the sum the longest path, i.e., $h_{T}(t) \vee h_{T}\left(t^{\prime}\right)$. Again, if the tree represents the behaviour of an agent in a synchronous setting, we get the maximum number of time units that may pass before our agent has to ask for new memory. If $T=\Sigma$ we get the usual definition of tree height, i.e., the maximum linear length of a path in the tree. 
Example 2.7 Let $T \subseteq \Sigma$. Call $b_{T}$ the unique morphism from $\mathbb{M}_{\Sigma}$ to $\left\langle\mathbb{N}, \vee, 0, f^{\prime}\right\rangle$, where $\sigma n=n+1 \forall \sigma \in T, \sigma n=n \forall \sigma \notin T$.

This map tells us the maximum number of arcs labelled in $T$ one can pass through going from the root to a leaf, i.e., how many times at best a desired action (or set of actions) will be performed. Notice that $h_{\Sigma}=b_{\Sigma}$.

Example 2.8 Let $T \subseteq \Sigma$. Call $w_{T}$ the unique morphism from $\mathbb{M}_{\Sigma}$ to $\left\langle\mathbb{N}, \dot{\Lambda}, 0, f^{\prime}\right\rangle$, where $\sigma n=n+1 \forall \sigma \in T, \sigma n=n \forall \sigma \notin T$.

This map tell us the minimum number of arcs labelled in $T$ one can pass through going from the root to a leaf, i.e., how many times at worst a desired action (or set of actions) will be performed.

Example 2.9 Let $T \subseteq \Sigma$. Call $m_{T}$ the unique morphism from $\mathbb{M}_{\Sigma}$ to $\left\langle\mathbb{N}, \dot{\wedge}, 0, f^{\prime}\right\rangle$, where $\sigma n=n+1 \forall \sigma \in T, \sigma n=0 \forall \sigma \notin T$.

This map counts the number of arcs one passes through starting from the root (regardless the path) before reaching a label out of $T$. Overworking a bit our agent example, this map tells us how many time units must pass before some new memory is needed. If $T=\Sigma$, we get the length of the shortest path available. Notice that $w_{\Sigma}=m_{\Sigma}$.

\subsection{Morphisms to $\Sigma^{*}$}

We want to extend some of the examples of the previous section, or, better, to refine them. We defined several counting maps which provide a quantitative evaluation of some properties of trees in $\mathbf{M}_{\Sigma}$. It is interesting to have also some kind of qualitative evaluations, and this is the aim of this section. We will use (with a slight abuse of notation) the same letters as before, for a reason that will be made clear in a few lines. We will write - for the concatenation in $\Sigma^{*}, \varepsilon$ for the empty word and $x$ for an arbitrary element of $\Sigma^{*}$.

Example 2.10 Let $T \subseteq \Sigma$. Call $\nu_{T}$ the unique morphism from $\mathbb{M}_{\Sigma}$ to $\left\langle\Sigma^{*}, \cdot, \varepsilon, f^{\prime}\right\rangle$, where $\sigma x=\sigma \cdot x \forall \sigma \in T, \sigma x=x \forall \sigma \notin T$.

$\nu_{T}$ produces the ordered list of the arcs we would meet if we scanned completely a tree with the preorder traversal (using the usual recursive algorithm) [Knu73]. However, only labels in $T$ would appear in the list. Note that simply by inverting the concatenation (i.e., $\sigma x=x \cdot \sigma \forall \sigma \in T$ ) we could have got the postorder traversal [Knu73].

Example 2.11 Let $T \subseteq \Sigma$. Call $\partial_{0}^{T}$ the unique morphism from $\mathbb{M}_{\Sigma}$ to $\left\langle\Sigma^{*}, \cdot, \varepsilon, f^{\prime}\right\rangle$, where $\sigma x=x \forall \sigma \in T, x \neq \varepsilon, \sigma \varepsilon=\sigma \forall \sigma \in T, \sigma x=x \forall \sigma \notin T$.

$\partial_{0}^{T}$ lists the arcs with labels in $T$ which are not followed (at any depth) by other arcs still in $T$. In other words, this is the frontier of $T . \partial_{0}$ lists the label of all the terminal arcs.

Example 2.12 Let $T \subseteq \Sigma$. Call $\partial_{1}^{T}$ the unique morphism from $\mathbb{M}_{\Sigma}$ to $\left\langle\Sigma^{*}, \cdot, \varepsilon, f^{\prime}\right\rangle$, where $\sigma x=\sigma \cdot x \forall \sigma \in T, \sigma x=\varepsilon \forall \sigma \notin T$. 
$\partial_{1}^{T}$ lists the labels in $T$ of the initial arcs (arcs out of the root). The names $\partial_{0}$ and $\partial_{1}$ come from the fact that a tree $t$ is, in a sense, a map with domain $\partial_{0}(t)$ and codomain $\partial_{1}(t)$.

Example 2.13 Let $T \subseteq \Sigma$. Call $\mu_{T}$ the unique morphism from $\mathbb{M}_{\Sigma}$ to $\left\langle\Sigma^{*}, \cdot, \varepsilon, f^{\prime}\right\rangle$, where $\sigma x=x \forall \sigma \in T, x \neq \varepsilon, \sigma \varepsilon=\sigma \forall \sigma \in T, \sigma x=\varepsilon \forall \sigma \notin T$.

$\mu_{T}$ lists the labels of the arcs which are not followed by other actions in $T$ reachable by passing only through actions in $T$.

Example 2.14 Let $T \subseteq \Sigma$. Call $\rho_{T}$ the unique morphism from $\mathbb{M}_{\Sigma}$ to $\left\langle\Sigma^{*}, \cdot, \varepsilon, f^{\prime}\right\rangle$, where $\sigma x=\sigma \cdot x \forall \sigma \in T, \sigma x=\varepsilon \forall \sigma \notin T$.

Finally, this map does a "preorder traversal" like $\nu$, but stops as soon as an action out of $T$ is met, rather than getting always down to the leaves.

Now, the map $|-|$ (length of a word) is a monoid morphism from $\left\langle\Sigma^{*}, \cdot, \varepsilon\right\rangle$ to $\langle\mathbb{N},+, 0\rangle$. But the relevant thing is that it is also a $\Sigma$-motor morphism if we take for $\Sigma^{*}$ and $\mathbb{N}$ the right actions. For instance, in defining the map $\nu$ in this section we used the product by $\sigma$ on the left for elements in $T$ and the identity out of $T$. Earlier, in the analogous definition of the previous section, we took $s: \mathbb{N} \longrightarrow \mathbb{N}$ in $T$ and the identity out of $T$ : it's obvious that $|\sigma \cdot(-)|=s(|-|)$. Thus,

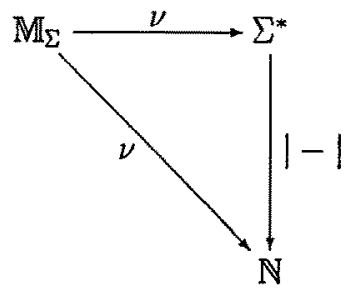

commutes, because $\mathbb{M}_{\Sigma}$ is initial. So, all the maps with the same name in the previous section differ by the ones we just defined "only" for a composition with $|-|$. Through $1-1$, we abstract from the qualitative characterization of this section, and we get a number instead of a list of labels (actions). In the following, the context will always make clear what definition we're using.

We must also account for the limitations of our machinery. In fact, there are still few interesting concept that we can't express: for example, how many terminal arcs have labels in $T$ ? The problem is that this can not be made into a $\Sigma$-motor map (guess why). But we can work around this difficulty considering arrows in Mon, that are anyway well behaved. We have only to use suitable "erasing maps" which delete from a string the labels we don't want.

We conclude by observing that this section deals with non-abelian $\Sigma$-motors. Obviously, everything can be restated using the free abelian monoid on $\Sigma$. 


\section{Algebraic properties}

This section is devoted to the basic algebraic properties of $\mathbb{M}_{\Sigma}$. The invariant description of Section 2 does not seem to provide any tool for direct manipulation of trees. This could give the impression that "invariant means unfriendly to handle". On the contrary, through a unique decomposition theorem (which extends Bénabou's result about unlabelled forests) we will link the invariant definition to a manageable representation: every tree $t \in \mathbb{M}_{\Sigma}$ will be uniquely writable as $\sigma_{1} x_{1} \oplus \sigma_{2} x_{2} \oplus \cdots \oplus \sigma_{n} x_{n}$, i.e., as a precise number of subtrees $x_{1}, x_{2}, \ldots, x_{n}$ linked to the root through arcs labelled respectively by $\sigma_{1}, \sigma_{2}, \ldots, \sigma_{n}$. We will see that definitions and proofs about $\mathbb{M}_{\Sigma}$ can be entirely given by induction, a property directly depending on initiality.

We will deal with the adjunction $(-)^{*} \dashv U$, where $(-)^{*}$ : Sets $\rightarrow$ Mon is the free monoid functor and $U:$ Mon $\rightarrow$ Sets is the forgetful functor. We will write $\phi$ for the natural bijection of morphisms, i.e., $\phi: \operatorname{Sets}(S, U(M)) \longrightarrow \operatorname{Mon}\left(S^{*}, M\right)$. The basic tools for doing computations will be the naturality conditions $\eta \circ k=U\left(k^{*}\right) \circ \eta$, $\phi(h \circ k)=\phi(h) \circ k^{*}$ and $\phi(U(h) \circ k)=h \circ \phi(k)$. The forgetful functor $\Sigma$-Mot $\longrightarrow$ Mon will be always understood.

\subsection{Unique decomposition}

Definition 3.1 Let $(\Sigma \times-)$ : Sets $\longrightarrow$ Sets be the "product by $\Sigma$ " functor (with the trivial action on morphisms). We define

$$
\Lambda_{\Sigma}=(-)^{*} \circ(\Sigma \times-) \circ U=(\Sigma \times U(-))^{*}: \text { Mon } \longrightarrow \text { Mon }
$$

Let us give a short description of how $\Lambda_{\Sigma}$ works. It takes a monoid $M$ to the free monoid generated by the elements of the set $\Sigma \times U(M)$, i.e., to the set of strings of the form

$$
\left\langle\sigma_{1}, x_{1}\right\rangle\left\langle\sigma_{2}, x_{2}\right\rangle \cdots\left\langle\sigma_{n}, x_{n}\right\rangle
$$

where $n$ varies in $\mathbb{N}, x_{i} \in M, \sigma_{i} \in \Sigma$.

The intuitive meaning of this operation can be understood if we apply it to $\mathbb{M}_{\Sigma}$ : in this case, we should think of the $x_{i}$ 's as trees which are copied in $|\Sigma|$ different ways and then freely concatenated: our interest will be in showing that this construction leads again to $\mathbb{M}_{\Sigma}$.

Now, we want to extend $\Lambda_{\Sigma}$ to a functor $\Sigma$-Mot $\longrightarrow \Sigma$-Mot. We will still use the same name $\Lambda_{\Sigma}$, and the context should make clear where we're working.

Proposition 3.1 $\Lambda_{\Sigma}$ can be made into a functor from $\Sigma$-Mot to $\Sigma$-Mot by composition with the forgetful functor $\Sigma$-Mot $\longrightarrow$ Mon, and by endowing the monoid $\Lambda_{\Sigma}(M)$ with the map

$$
\eta \circ\left(\mathbf{1}_{\Sigma} \times U(\phi(f))\right)
$$

where $\eta$ is the insertion of generators

$$
\eta: \Sigma \times U(M) \longrightarrow U\left((\Sigma \times U(M))^{*}\right)
$$

and $f$ is the action of $M$. 
In terms of elements, the map $\eta \circ\left(1_{\Sigma} \times U(\phi(f))\right)$, when applied to a label $\sigma$ and to a string $\left\langle\sigma_{1}, x_{1}\right\rangle\left\langle\sigma_{2}, x_{2}\right\rangle \cdots\left\langle\sigma_{n}, x_{n}\right\rangle$, produces the one-character string

$$
\left\langle\sigma, \sigma_{1} x_{1} \oplus \sigma_{2} x_{2} \oplus \cdots \oplus \sigma_{n} x_{n}\right\rangle
$$

Proof. We must only prove $\Lambda_{\Sigma}$ takes arrows of $\Sigma$-Mot into arrows of $\Sigma$-Mot. Let

$$
A:\langle M, \oplus, 0, f\rangle \longrightarrow\left\langle M^{\prime}, \oplus, 0, g\right\rangle
$$

be an arrow in $\Sigma$-Mot; then $\Lambda_{\Sigma}(A)$ is trivially a monoid map, and

$$
\begin{aligned}
U\left(\Lambda_{\Sigma}(A)\right) \circ f^{\prime} & =U\left(\Lambda_{\Sigma}(A)\right) \circ \eta \circ\left(\mathbf{1}_{\Sigma} \times U(\phi(f))\right) \\
& =\eta \circ\left(\mathbf{1}_{\Sigma} \times U(A)\right) \circ\left(\mathbf{1}_{\Sigma} \times U(\phi(f))\right) \\
& =\eta \circ\left(\mathbf{1}_{\Sigma} \times U(A \circ \phi(f))\right) \\
& =\eta \circ\left(\mathbf{1}_{\Sigma} \times U(\phi(U(A) \circ f))\right) \\
& =\eta \circ\left(\mathbf{1}_{\Sigma} \times U\left(\phi\left(g \circ\left(\mathbf{1}_{\Sigma} \times U(A)\right)\right)\right)\right) \\
& =\eta \circ\left(\mathbf{1}_{\Sigma} \times U\left(\phi(g) \circ\left(\mathbf{1}_{\Sigma} \times U(A)\right)^{*}\right)\right) \\
& =\eta \circ\left(\mathbf{1}_{\Sigma} \times U(\phi(g))\right) \circ\left(\mathbf{1}_{\Sigma} \times U\left(\Lambda_{\Sigma}(A)\right)\right) \\
& =g^{\prime} \circ\left(\mathbf{1}_{\Sigma} \times U\left(\Lambda_{\Sigma}(A)\right)\right)
\end{aligned}
$$

We are now able to prove the following

Theorem $3.1 \quad \mathbb{M}_{\Sigma} \cong \Lambda_{\Sigma}\left(\mathbb{M}_{\Sigma}\right)$.

Proof. We have of course a unique morphism

$$
\mathbb{M}_{\Sigma} \stackrel{D}{\longrightarrow} \Lambda_{\Sigma}\left(\mathbb{M}_{\Sigma}\right)
$$

To build an inverse, take the map $E:\left(\Sigma \times U\left(\mathbb{M}_{\Sigma}\right)\right)^{*} \longrightarrow \mathbb{M}_{\Sigma}$ which corresponds to $f$ in the adjunction $(-)^{*} \dashv U$, i.e., $E=\phi(f) . E$ is a $\Sigma$-motor map, because

$$
U(E) \circ f^{\prime}=U(\phi(f)) \circ \eta \circ\left(1_{\Sigma} \times U(\phi(f))\right)=f \circ\left(\mathbf{1}_{\Sigma} \times U(E)\right) .
$$

Now, $E \circ D=\mathbf{1}_{\mathbb{M}_{\Sigma}}$ by initiality of $\mathbb{M}_{\Sigma}$, and we have

$$
\begin{aligned}
D \circ E & =\phi(U(D) \circ f) \\
& =\phi\left(f^{\prime} \circ\left(\mathbf{1}_{\Sigma} \times U(D)\right)\right) \\
& =\phi\left(\eta \circ\left(\mathbf{1}_{\Sigma} \times U(\phi(f))\right) \circ\left(\mathbf{1}_{\Sigma} \times U(D)\right)\right) \\
& =\phi\left(\left(\left(\mathbf{1}_{\Sigma} \times U(E)\right) \circ\left(\mathbf{1}_{\Sigma} \times U(D)\right)\right)^{*} \circ \eta\right) \\
& =\left(\mathbf{1}_{\Sigma} \times U(E \circ D)\right)^{*} \\
& =\left(\mathbf{1}_{\Sigma} \times U\left(\mathbf{1}_{\mathbf{M}_{\Sigma}}\right)\right)^{*} \\
& =\mathbf{1}_{\Lambda_{\Sigma}\left(M_{\Sigma}\right)}
\end{aligned}
$$

and this proves that $\mathbb{M}_{\Sigma} \cong \Lambda_{\Sigma}\left(\mathbb{M}_{\Sigma}\right)$. 
Corollary 3.1 Every tree $t \in \mathbb{M}_{\Sigma}$ has a unique decomposition $\sigma_{1} x_{1} \oplus \sigma_{2} x_{2} \oplus \cdots \oplus \sigma_{n} x_{n}$.

$$
\begin{aligned}
t & =E(D(t)) \\
& =E\left(\left\langle\sigma_{1}, x_{1}\right\rangle\left\langle\sigma_{2}, x_{2}\right\rangle \cdots\left\langle\sigma_{n}, x_{n}\right\rangle\right) \\
& =\sigma_{1} x_{1} \oplus \sigma_{2} x_{2} \oplus \cdots \oplus \sigma_{n} x_{n}
\end{aligned}
$$

The uniqueness is trivial because $D$ is an isomorphism.

Corollary 3.2 Let $t \in \mathbb{M}_{\Sigma}, t=\sigma_{1} x_{1} \oplus \sigma_{2} x_{2} \oplus \cdots \oplus \sigma_{n} x_{n}$. Then, $\nu(t)=0$ iff $t=0$ and, if $n>0, \nu\left(x_{k}\right)<\nu(t) \forall k$.

$$
\begin{aligned}
\nu(t) & =\nu\left(\sigma_{1} x_{1} \oplus \sigma_{2} x_{2} \oplus \cdots \oplus \sigma_{n} x_{n}\right) \\
& =\nu\left(\sigma_{1} x_{1}\right)+\nu\left(\sigma_{2} x_{2}\right)+\cdots+\nu\left(\sigma_{n} x_{n}\right) \\
& =s\left(\nu\left(x_{1}\right)\right)+s\left(\nu\left(x_{2}\right)\right)+\cdots+s\left(\nu\left(x_{n}\right)\right) \\
& =n+\nu\left(x_{1}\right)+\nu\left(x_{2}\right)+\cdots+\nu\left(x_{n}\right)
\end{aligned}
$$

Thus, if $n>0, \nu\left(x_{k}\right)<\nu(t) \forall k$, and if $n=0, t=0$.

This corollary shows clearly the recursive nature of $\mathbb{M}_{\Sigma}$, and enables us to use induction on the number of arcs to give proofs and definitions, as it is usual both in theoretical and practical programming.

Induction can also be directly derived from the universal property of $\mathbb{M}_{\Sigma}$, much in the same way as induction on natural numbers is a direct consequence of the Peano-Lawvere axiom (see for instance [MB67]). Indeed, suppose we have a subset $P \subseteq U\left(\mathbb{M}_{\Sigma}\right)$ such that $0 \in P$, and $\forall x, y \in P \forall \sigma \in \Sigma$ we have that $x \oplus y \in P$ and $\sigma x \in P$. Then, $\langle P, \oplus, 0, f\rangle$ (where $\oplus$ and 'f are restricted in the obvious way) is a $\Sigma$-motor, so we have a unique morphism

$$
\mathbb{M}_{\Sigma} \stackrel{!_{P}}{\longrightarrow} P
$$

in $\Sigma$-Mot. But the inclusion $j: P \longrightarrow U\left(\mathbb{M}_{\Sigma}\right)$ is trivially a $\Sigma$-motor map, so we have $j \circ !_{P}=\mathbf{1}_{\mathbf{M}_{\Sigma}}$. This implies $j$ is epi. Since $j$ is also a mono, $j$ is an iso, i.e., $P=U\left(\mathbb{M}_{\Sigma}\right)$.

Thus, initiality and induction are essentially two sides of the same medal. The choice of which one to use is essentially a matter of convenience. For instance, the last computation in the proof of Theorem 3.1 could have been done using induction. Instead, using only arrows we worked out the expression until $E \circ D$ appeared, and this map is an identity by initiality of $\mathbb{M}_{\Sigma}$.

Note also that in view of Corollary 3.2 , instead of proving

$$
0 \in P \wedge \forall x, y \in P \forall \sigma \in \Sigma(x \oplus y \in P \wedge \sigma x \in P)
$$

one can prove for an arbitrary natural number $n$

$$
\forall x_{1}, x_{2}, \ldots, x_{n} \in P \forall \sigma_{1}, \sigma_{2}, \ldots, \sigma_{n} \in \Sigma\left(\sigma_{1} x_{1} \oplus \sigma_{2} x_{2} \oplus \cdots \oplus \sigma_{n} x_{n} \in P\right) .
$$




\section{2 $\mathbb{M}_{\Sigma}$ as a colimit}

Theorem 3.2 Consider the functor $F: \mathbb{N} \longrightarrow \operatorname{Mon},(\mathbb{N}$ is thought of as an ordered set)

$$
F(k)=\Lambda_{\Sigma}{ }^{k}(1)
$$

which to every arrow $i_{n}=n \leq(n+1)$ assigns

$$
\begin{aligned}
& F\left(i_{0}\right)=1 \stackrel{\Sigma_{\Sigma *}^{*}}{\longrightarrow} \Sigma^{*} \\
& F\left(i_{n}\right)=\Lambda_{\Sigma}\left(F\left(i_{n-1}\right)\right)
\end{aligned}
$$

Then $\lim _{\longrightarrow} F$ endowed with a suitable $f^{\prime}$ is isomorphic to $\mathbb{M}_{\Sigma}$ in $\Sigma$-Mot.

Proof. Since $\Sigma \times U\left(\lim _{\rightarrow} F\right) \cong \lim _{\rightarrow} \Sigma \times U(F)$, we define the $n$th component of $f^{\prime}$ on $\underset{\lim }{\longrightarrow} \times U(F)$ as

$$
f_{n}^{\prime}=U\left(\mu_{n+1}\right) \circ \eta: \Sigma \times U(F(n)) \longrightarrow U\left((\Sigma \times U(F(n)))^{*}\right)=U(F(n+1)) \longrightarrow U(\underline{\lim } F)
$$

This way, we get a (unique) map ! : $\mathbb{M}_{\Sigma} \longrightarrow \lim F$. As for the inverse, we define it directly in Mon, and then we prove that it is in fact in $\Sigma$-Mot. Let

$$
\begin{aligned}
\psi_{0} & =1 \longrightarrow \mathbb{M}_{\Sigma} \\
\psi_{n} & =E \circ \Lambda_{\Sigma}\left(\psi_{n-1}\right): F(n) \longrightarrow \mathbb{M}_{\Sigma}
\end{aligned}
$$

Now, $\left\{\psi_{n}\right\}$ defines a map in Mon, because by induction the following cones commute:

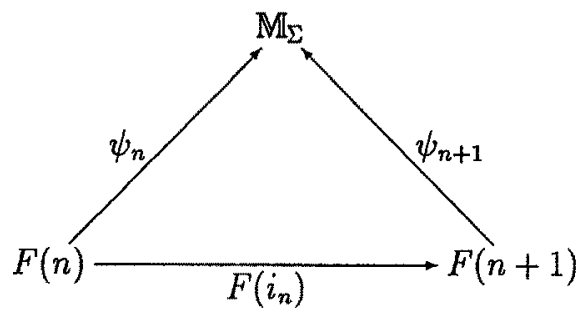

In fact,

$$
\begin{aligned}
\psi_{n+1} \circ F\left(i_{n}\right) & =E \circ \Lambda_{\Sigma}\left(\psi_{n}\right) \circ \Lambda_{\Sigma}\left(F\left(i_{n-1}\right)\right) \\
& =E \circ \Lambda_{\Sigma}\left(\psi_{n} \circ F\left(i_{n-1}\right)\right) \\
& =E \circ \Lambda_{\Sigma}\left(\psi_{n-1}\right) \\
& =\psi_{n}
\end{aligned}
$$

and the first cone

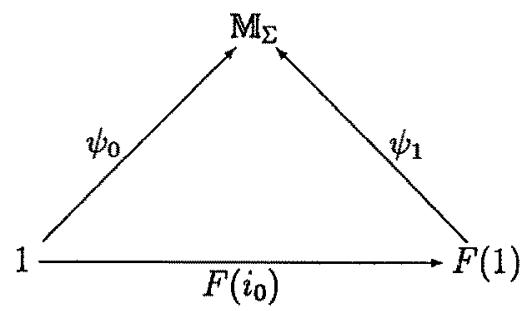


commutes since the starting vertex is 1 . But $\psi$ (defined on the colimit through the $\psi_{n}$ 's) is also an arrow in $\Sigma$-Mot:

$$
\begin{aligned}
U(\psi) \circ U\left(\mu_{n+1}\right) \circ \eta & =U\left(\psi_{n+1}\right) \circ \eta \\
& =U\left(E \circ \Lambda_{\Sigma}\left(\psi_{n}\right)\right) \circ \eta \\
& =U(E) \circ U\left(\Lambda_{\Sigma}\left(\psi_{n}\right)\right) \circ \eta \\
& =U(E) \circ \eta \circ\left(\mathbf{1}_{\Sigma} \times U\left(\psi_{n}\right)\right) \\
& =f \circ\left(\mathbf{1}_{\Sigma} \times U\left(\psi_{n}\right)\right)
\end{aligned}
$$

so that we have $\psi \circ !=\mathbf{1}_{\mathbf{M}_{2}}$. Now, $! \circ \psi_{0}=\mu_{0}$ trivially, and by induction

$$
\begin{aligned}
1 \circ \psi_{n} & =! \circ E \circ \Lambda_{\Sigma}\left(\psi_{n-1}\right) \\
& =! \circ \phi\left(f \circ\left(\mathbf{1}_{\Sigma} \times U\left(\psi_{n-1}\right)\right)\right) \\
& =\phi\left(U(!) \circ f \circ\left(\mathbf{1}_{\Sigma} \times U\left(\psi_{n-1}\right)\right)\right) \\
& =\phi\left(f^{\prime} \circ\left(\mathbf{1}_{\Sigma} \times U(!) \circ U\left(\psi_{n-1}\right)\right)\right) \\
& =\phi\left(f^{\prime} \circ\left(\mathbf{1}_{\Sigma} \times U\left(! \circ \psi_{n-1}\right)\right)\right) \\
& =\phi\left(f^{\prime} \circ\left(\mathbf{1}_{\Sigma} \times U\left(\mu_{n-1}\right)\right)\right) \\
& =\phi\left(f_{n-1}^{\prime}\right) \\
& =\phi\left(U\left(\mu_{n}\right) \circ \eta\right) \\
& =\mu_{n}
\end{aligned}
$$

Thus,,$\circ \psi=\mathbf{1}_{\underline{l i m} F}$.

\section{3 $\mathbb{M}_{\Sigma}$ as a pullback}

There is also another interesting characterization of $\mathbb{M}_{\Sigma}$, due to Bénabou.

Theorem 3.3 (Bénabou) Consider the following $\Sigma$-motors: $\Sigma^{*}$ with $\sigma x=\sigma \cdot x,\langle\mathbb{N},+, 0, s\rangle$ and $\langle\mathbb{M}, \oplus, 0, f\rangle$. Then $\mathbb{M}_{\Sigma}$ is the pullback in $\Sigma-\operatorname{Mot}$ of $|-|: \Sigma^{*} \longrightarrow \mathbb{N}$ and $\nu: \mathbb{M} \longrightarrow \mathbb{N}$.

The proposition shows that, in a sense, $\mathbb{M}_{\Sigma}$ is a labelled version of $\mathbb{M}$. We get the following pullback diagram:

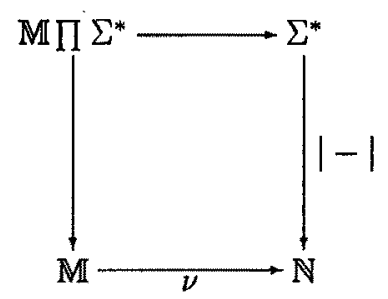

The pullback is formed by pairs $(t, x)$ with $t \in \mathbb{M}, x \in \Sigma^{*}$ and $|x|=\nu(t)$, and the unique map

$$
\mathbb{M}_{\Sigma} \stackrel{!}{\rightarrow} \mathbb{M} \prod \Sigma^{*}
$$

turns out to be an isomorphism. For the details of the proof, see [Bén90]. 


\subsection{Parallel translation}

We will see in this section that $\mathbb{M}_{\Sigma}$ has an interesting property which will allow us to freely extend any map $\Sigma \times U\left(\mathbb{M}_{\Sigma}\right) \longrightarrow M$ to a monoid morphism, in analogy with Bénabou's corresponding notion for motors.

Theorem 3.4 Given a motor $\left\langle M, \oplus, 0, f^{\prime}\right\rangle$ and a map $u: \Sigma \times U\left(\mathbb{M}_{\Sigma}\right) \longrightarrow M$ there is a unique arrow $\mathcal{T}(u)$ in Mon such that the following diagram commutes:

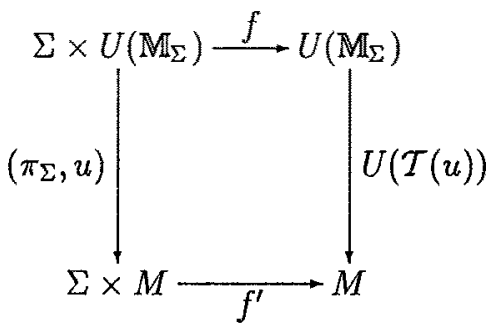

where $\pi_{\Sigma}: \Sigma \times U\left(\mathbb{M}_{\Sigma}\right) \longrightarrow \Sigma$ is the canonical projection, i.e., $U(\mathcal{T}(u)) \circ \sigma=\sigma \circ u(\sigma,-)$ for each $\sigma \in \Sigma$.

Proof. The map $\mathcal{T}(u)$ is defined through universal properties, namely we take the map corresponding to $f^{\prime} \circ\left(\pi_{\Sigma}, u\right)$ in the adjunction $(-)^{*} \dashv U(-)$ and we compose it with the isomorphism $D$

$$
\mathcal{T}(u)=\phi\left(f^{\prime} \circ\left(\pi_{\Sigma}, u\right)\right) \circ D: \mathbb{M}_{\Sigma} \longrightarrow M
$$

And, what matters,

$$
\begin{aligned}
U(\mathcal{T}(u)) \circ f & =U\left(\phi\left(f^{\prime} \circ\left(\pi_{\Sigma}, u\right)\right)\right) \circ U(D) \circ f \\
& =U\left(\phi\left(f^{\prime} \circ\left(\pi_{\Sigma}, u\right)\right)\right) \circ f^{\prime} \circ\left(\mathbf{1}_{\Sigma} \times U(D)\right) \\
& =U\left(\phi\left(f^{\prime} \circ\left(\pi_{\Sigma}, u\right)\right)\right) \circ \eta \circ\left(\mathbf{1}_{\Sigma} \times U(\phi(f)) \circ\left(\mathbf{1}_{\Sigma} \times U(D)\right)\right. \\
& =f^{\prime} \circ\left(\pi_{\Sigma}, u\right) \circ\left(\mathbf{1}_{\Sigma} \times U\left(\mathbf{1}_{\mathbf{M}_{\Sigma}}\right)\right) \\
& =f^{\prime} \circ\left(\pi_{\Sigma}, u\right)
\end{aligned}
$$

Finally, the arrow $\mathcal{T}(u)$ is unique because $U(D) \circ f$ is just the insertion of generators from $\Sigma \times U\left(\mathbb{M}_{\Sigma}\right)$ to $\Lambda_{\Sigma}\left(\mathbb{M}_{\Sigma}\right)$.

Often we will use a map $u: U\left(\mathbb{M}_{\Sigma}\right) \longrightarrow M$, and it is clear that the theorem can be applied to such a map, by composing it with the projection $\pi_{U\left(\mathbb{M}_{\Sigma}\right)}: \Sigma \times U\left(\mathbb{M}_{\Sigma}\right) \longrightarrow$ $U\left(\mathbf{M}_{\Sigma}\right)$.

It is useful to have an insight about parallel translation. The slogan here is "enter one level into the tree, apply $u$ and rebuild the tree with $f^{\prime \prime}$. Of course, the effect of $u$ depends on the label met while entering.

\section{Operation on trees}

We will now define, always using universal properties, several operation on trees, namely pruning, shortening, relabelling and scanning. Looking at their definitions, the intuition behind the "initial object method" as stated in Section 2.2 becomes much clearer. 


\subsection{Pruning}

The pruning of a tree is the abstract counterpart of the corresponding botanic operation: we want to cut out every branch starting with a label in a given subset $T$. The most common example is the restriction operator of CCS-like process description languages. An informal example is given by the following picture:

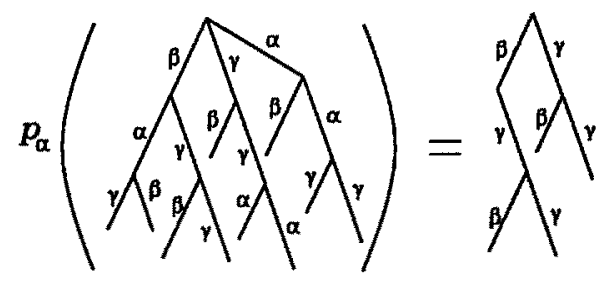

Definition 4.1 Let $T \subseteq \Sigma$. We call $p_{T}$ the unique morphism in $\Sigma$-Mot from $\mathbb{M}_{\Sigma}$ to $\mathbb{M}^{\prime}=\left\langle\mathbb{M}_{\Sigma \backslash T}, \oplus, 0, f^{\prime}\right\rangle$, where $\mathbb{M}_{\Sigma \backslash T}$ is the initial $\Sigma \backslash T$-motor, $\oplus$ its monoid operation, and $f^{\prime}(\sigma,-)=0 \forall \sigma \in T, f^{\prime}(\sigma,-)=\sigma \forall \sigma \notin T$.

We observe that $\mathbb{M}_{\Sigma \mid T}$ is endowed with a unique "injection morphism" $i_{T}$ in $\mathbb{M}_{\Sigma}$ (you need only to restrict $f$ to $\Sigma \backslash T$ ). And, what really matters, by the universality of $\mathbb{M}_{\Sigma \backslash T}$ in $\Sigma \backslash T$-Mot we have the following commutative diagram:

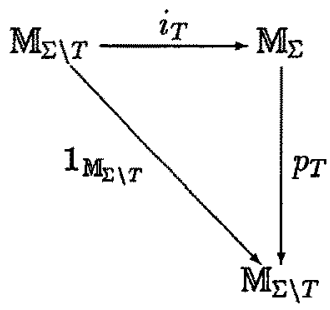

i.e., $p_{T} \circ i_{T}=\mathbf{1}_{M_{\Sigma \backslash T}}$.

The other interesting property is expressed by the following diagram:

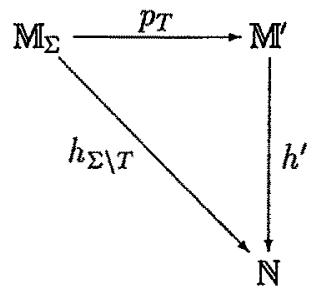

where $h^{\prime}$ is the height morphism in $\Sigma \backslash T$-Mot, that turns out to be a $\Sigma$-Mot morphism, too (in the definition, we have zero maps in $\Sigma \backslash T$ for both $\mathbb{N}$ and $\mathbb{M}^{\prime}$ ). The meaning is clear: since $h_{\Sigma \backslash T}$ counts the height stopping at the first label out of $T$, cutting away everything that is in $T$ doesn't change its value. The same thing is true for the map $m$, the "traversal" $\rho$, the map $\mu$, and the map $\partial_{1}$. 


\subsection{Shortening}

This notion has a strict relation with pruning, but instead of cutting out the branches we don't like, we simply shrink them, making them disappear. Again, we make a graphic example:

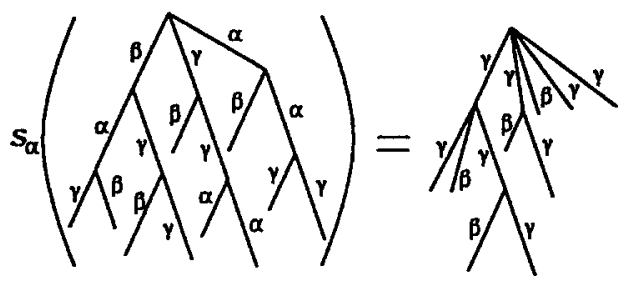

Definition 4.2 Let $T \subseteq \Sigma$. We call $s_{T}$ the unique morphism in $\Sigma$-Mot from $\mathbb{M}_{\Sigma}$ to $\mathbb{M}^{\prime}=\left\langle\mathbb{M}_{\Sigma \backslash T}, \oplus, 0, f^{\prime}\right\rangle$, where $\mathbb{M}_{\Sigma \backslash T}$ is the free $\Sigma \backslash T$-motor, $\oplus$ its monoid operation, and $f^{\prime}(\sigma,-)=\mathbf{1}_{\mathbb{M}_{\Sigma \backslash T}} \forall \sigma \in T, f^{\prime}(\sigma,-)=\sigma \forall \sigma \notin T$.

Again, $\mathbb{M}_{\Sigma \backslash T}$ has a unique injection morphism $i_{T}$ in $\mathbb{M}_{\Sigma}$, and in $\mathbf{M o t}_{\Sigma \backslash T}$ we have the following commutative diagram:

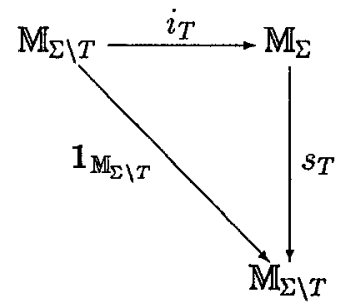

i.e., $s_{T} \circ i_{T}=\mathbf{1}_{\mathbf{M}_{\Sigma \backslash T}}$. We have preservation properties similar to pruning, but with different maps $\left(c, w, \nu, \partial_{0}\right)$.

\subsection{Relabelling}

We have another interesting operation on trees, namely relabelling. Let $\Sigma$ and $\Sigma^{\prime}$ be two alphabets. Then,

Definition 4.3 Given a relabelling map $\xi: \Sigma \longrightarrow \Sigma^{\prime}$, the relabelling arrow $R_{\xi}$ is the unique morphism in $\Sigma$-Mot from $\mathbb{M}_{\Sigma}$ to $\mathbb{M}^{\prime}=\left\langle\mathbb{M}_{\Sigma^{\prime}}, \oplus, 0, f^{\prime}(\xi(-),-)\right\rangle$.

We observe that we have an unlabelling arrow associated to the unique map in Sets $!: \Sigma \longrightarrow\{*\}$ that maps $\mathbb{M}_{\Sigma}$ in $\mathbb{M}$, and that the arc-counting map of $\mathbb{M}$ is an arrow in $\Sigma$-Mot; this implies that the following diagram commutes:

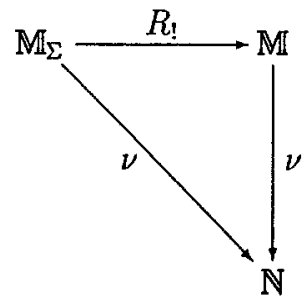


The fundamental property of relabelling is expressed by the next

Proposition 4.1 Let $\xi: \Sigma \longrightarrow \Sigma^{\prime}$ be a relabelling map, $R_{\xi}$ the relabelling arrow and $\xi^{*}: \Sigma^{*} \longrightarrow \Sigma^{\prime *}$ the image of $\xi$ through the free monoid functor. Given on $\Sigma^{*}$ and $\Sigma^{\prime *}$ two $\Sigma$-motor structures through maps $g$ and $g^{\prime}$ such that the following diagram commutes

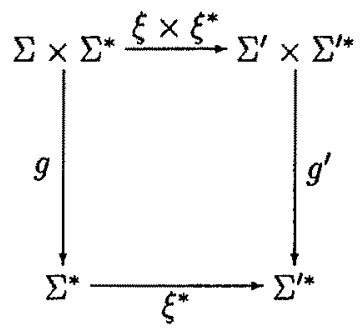

and called!,!' the corresponding unique maps $\mathbb{M}_{\Sigma} \stackrel{!}{\longrightarrow} \Sigma^{*}, \mathbb{M}_{\Sigma^{\prime}} \stackrel{!^{\prime}}{\longrightarrow} \Sigma^{* *}$, then the following diagram commutes in $\Sigma$-Mot:

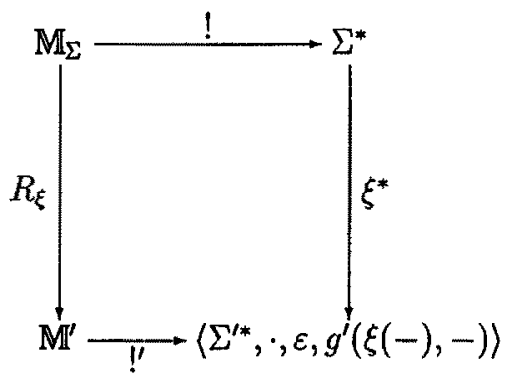

Besides, if $g(\sigma,-)=\sigma \cdot(-)$ and $g^{\prime}\left(\sigma^{\prime},-\right)=\sigma^{\prime} \cdot(-)$, i.e., $!=\nu$ and $!^{\prime}=\nu^{\prime}$, the diagram is a pullback.

We have only to prove !' is an arrow in $\Sigma$-Mot, since $\xi^{*}$ is in $\Sigma$-Mot by hypothesis. The monoid operation is trivially preserved,

$$
!^{\prime}\left(f^{\prime}(\xi(\sigma), t)\right)=g^{\prime}\left(\xi(\sigma), !^{\prime}(t)\right)
$$

and $g^{\prime}(\xi(-),-)$ is exactly the map for $\Sigma^{\prime *}$. The diagram then automatically commutes, since it starts from $\mathbb{M}_{\Sigma}$. The proof that in the case $!=\nu$ and $!^{\prime}=\nu^{\prime}$ the diagram is a pullback is straightforward.

The usefulness of this proposition relies on the fact that all the structures defined in the Section 2.2 and 2.3 in order to obtain meaningful morphisms by universal properties satisfy the commutation condition on $\xi^{*}$. That is, relabelling does not change the essential features of a tree-just passing through $\xi^{*}$ gives the "relabelled" result.

\subsection{Scanning}

Let us now define the scanning of a labelled tree following very closely Bénabou's notion for forests. The scanning of a tree is performed by a set of monoid maps $T_{n}$ and $B_{n}$ from $\mathrm{M}_{\Sigma}$ to $\mathbb{M}_{\Sigma} \cdot T_{n}(t)$ gives us the first $n$ "levels" of $t$, while $B_{n}$ tell us what remains of $t$ after $T_{n}(t)$ has been cut out. These maps, composed with other ones, can do a large number of operations from a purely algebraic viewpoint. They also give a good insight on how the parallel translation property works. 
Definition 4.4 Let $0: U\left(\mathbb{M}_{\Sigma}\right) \longrightarrow U\left(\mathbb{M}_{\Sigma}\right)$ be the zero constant map. The top scanning map $T_{n}$ is defined by

$$
T_{n}=\mathcal{T}^{n}(0)
$$

Definition 4.5 Let $1: U\left(\mathbb{M}_{\Sigma}\right) \longrightarrow U\left(\left\langle\mathbb{M}_{\Sigma}, \oplus, 0,1_{U\left(\mathbb{M}_{\Sigma}\right)}\right)\right)$ be the identity map. The bottom scanning map $B_{n}$ is defined by

$$
B_{n}=\mathcal{T}^{n}(\mathbf{1})
$$

Of course, definition of much more refined scanning maps is possible by simply changing the base function. For instance, if instead of the map 0 we used a map $u$ such that $u(\alpha, t)=0$ for some $\alpha \in \Sigma$ but $u(\beta, t)=t$ for all $\beta \neq \alpha$, the resulting map $\mathcal{T}^{n}(u)$ would cut at level $n$ only arcs starting with $\alpha$. More complex tasks can be carried on by making every step of the translation $\Sigma$-dependent.

\subsubsection{Properties of scanning}

We will now sketch some properties of the scanning maps, in order to get acquainted with the typical computational techniques of $\Sigma$-motors.

Proposition 4.2 Let $t \in \mathbb{M}_{\Sigma}, n, p \in \mathbb{N}, T \subseteq \Sigma$. Then

- (1) $h^{T}\left(T_{n}(t)\right)=n \wedge h^{T}(t)$

- (2) If $p \leq n, T_{p} \circ T_{n}=T_{n} \circ T_{p}=T_{p}$

- (3) If $h(t) \leq n, B_{n}(t)=0$

- (4) $h\left(T_{n}(t)\right)+h\left(B_{n}(t)\right)=h(t)$

- (5) If $0<n \leq h(t), \partial_{0}\left(T_{n}(t)\right)=\partial_{1}\left(B_{n-1}(t)\right)$

(1) For $n=0$ the property is trivial. But

$$
\begin{aligned}
h^{T}\left(T_{n}(t)\right) & =h^{T}\left(\mathcal{T}\left(T_{n-1}\right)\left(\sum_{k} \sigma_{k} x_{k}\right)\right) \\
& =h^{T}\left(\sum_{k} \sigma_{k}\left(T_{n-1}\left(x_{k}\right)\right)\right) \\
& \left.=\bigvee\left(h^{T}\left(T_{n-1}\left(x_{k}\right)\right)\right)+1\right) \\
& =\left(\bigvee_{k} h^{T}\left(T_{n-1}\left(x_{k}\right)\right)\right)+1 \\
& \left.=\underset{k}{(}(n-1) \wedge h^{T}\left(x_{k}\right)\right)+1 \\
& =(n-1) \wedge\left(\underset{k}{V} h^{T}\left(x_{k}\right)\right)+1 \\
& =n \wedge\left(\bigvee_{k}^{T} h^{T}\left(x_{k}\right)+1\right) \\
& =n \wedge h^{T}(t)
\end{aligned}
$$


$(2)$

$$
\begin{aligned}
T_{p} \circ T_{n} & =\mathcal{T}^{p}\left(T_{0}\right) \circ \mathcal{T}^{p}\left(T_{n-p}\right) \\
& =\mathcal{T}^{p}\left(T_{0} \circ T_{n-p}\right) \\
& =\mathcal{T}^{p}\left(T_{0}\right) \\
& =T_{p}
\end{aligned}
$$

(3)

$$
\begin{aligned}
h\left(B_{n}(t)\right) & =B_{n}\left(\sum_{k} \sigma_{k} x_{k}\right) \\
& =\sum_{j} B_{n-1}\left(x_{j}\right) \\
& =0
\end{aligned}
$$

since $h\left(x_{i}\right)<h(t) \leq n$.

(4)

$$
\begin{aligned}
h\left(T_{n}(t)\right)+h\left(B_{n}(t)\right) & =h\left(T_{n}\left(\sum_{k} \sigma_{k} x_{k}\right)\right)+h\left(B_{n}\left(\sum_{k} \sigma_{k} x_{k}\right)\right) \\
& =h\left(\sum_{k} \sigma_{k}\left(T_{n-1}\left(x_{k}\right)\right)\right)+h\left(\sum_{k} B_{n-1}\left(x_{k}\right)\right) \\
& =\bigvee_{k} s\left(h\left(T_{n-1}\left(x_{k}\right)\right)\right)+\bigvee_{k} h\left(B_{n-1}\left(x_{k}\right)\right) \\
& =\bigvee_{k}\left(s\left(h\left(T_{n-1}\left(x_{k}\right)\right)\right)+h\left(B_{n-1}\left(x_{k}\right)\right)\right) \\
& =\bigvee_{k} s\left(h\left(x_{k}\right)\right) \\
& =\bigvee_{k} h\left(\sigma_{k} x_{k}\right) \\
& =h(t)
\end{aligned}
$$

Notice that the passage from the third to the fourth line, in which we discard the crossed sups, i.e., sups of the form

$$
s\left(h\left(T_{n-1}\left(x_{i}\right)\right)\right)+h\left(B_{n-1}\left(x_{j}\right)\right) \quad \text { with } i \neq j,
$$

is justified by the following little argument: we distribute the sum on the sups in the third line, and we pick up the biggest addendum $s\left(h\left(T_{n-1}\left(x_{i}\right)\right)\right)+h\left(B_{n-1}\left(x_{j}\right)\right)$. Then $s\left(h\left(T_{n-1}\left(x_{i}\right)\right)\right) \leq n$ for the property (1). But $h\left(B_{n-1}\left(x_{j}\right)\right)>0$, for otherwise we could replace it with $h\left(B_{n-1}\left(x_{i}\right)\right)$. This implies $s\left(h\left(T_{n-1}\left(x_{j}\right)\right)\right)=n$, for property (3), so that

$$
s\left(h\left(T_{n-1}\left(x_{j}\right)\right)\right)+h\left(B_{n-1}\left(x_{j}\right)\right) \geq s\left(h\left(T_{n-1}\left(x_{i}\right)\right)\right)+h\left(B_{n-1}\left(x_{j}\right)\right) .
$$

(5) In this case, the base step is not so simple, so we work it out:

$$
\begin{aligned}
\partial_{0}\left(T_{1}(t)\right) & =\sum_{k} \partial_{0}\left(\sigma_{k}\left(T_{0}\left(x_{k}\right)\right)\right) \\
& =\partial_{0}\left(\sigma_{1}\right) \cdots \partial_{0}\left(\sigma_{n}\right) \\
& =\partial_{1}\left(\sigma_{1}\right) \cdots \partial_{1}\left(\sigma_{n}\right) \\
& =\partial_{1}\left(\sigma_{1} x_{1}\right) \cdots \partial_{n}\left(\sigma_{n} x_{n}\right) \\
& =\partial_{1}(t) \\
& =\partial_{1}\left(B_{0}(t)\right)
\end{aligned}
$$


Then,

$$
\begin{aligned}
\partial_{0}\left(T_{n}(t)\right) & =\partial_{0}\left(\sum_{k} \sigma_{k}\left(T_{n-1}\left(x_{k}\right)\right)\right) \\
& =\sigma_{1}\left(\partial_{0}\left(T_{n-1}\left(x_{1}\right)\right)\right) \cdots \sigma_{m}\left(\partial_{0}\left(T_{n-1}\left(x_{m}\right)\right)\right) \\
& =\sigma_{1}\left(\partial_{1}\left(B_{n-2}\left(x_{1}\right)\right)\right) \cdots \sigma_{m}\left(\partial_{1}\left(B_{n-2}\left(x_{m}\right)\right)\right) \\
& =\sigma_{1}\left(\partial_{1}\left(B_{n-1}\left(\sigma_{1} x_{1}\right)\right)\right) \cdots \sigma_{m}\left(\partial_{1}\left(B_{n-1}\left(\sigma_{m} x_{m}\right)\right)\right) \\
& =\partial_{1}\left(B_{n-1}\left(\sigma_{1} x_{1}\right)\right) \cdots \partial_{1}\left(B_{n-1}\left(\sigma_{m} x_{m}\right)\right) \\
& =\partial_{1}\left(B_{n-1}(t)\right)
\end{aligned}
$$

\section{Binary operations on trees}

In this section we will study some binary operations on trees.

\subsection{Tree product}

An important operation on trees consists in attaching a copy of a given tree to each node of another tree, as in the following picture:

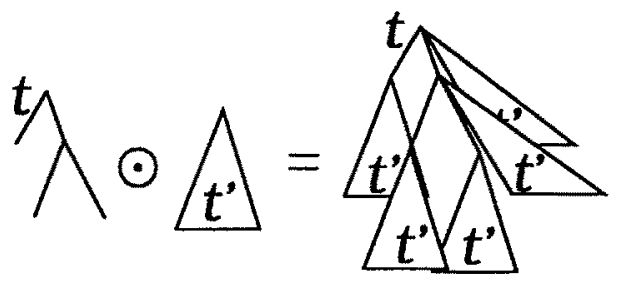

We note that one can choose to attach the tree to the left or to the right of the arcs already existing under the node, and this leads to two slightly different operations. In an abelian setting, of course, this does not happen.

Definition 5.1 Let $t, t^{\prime} \in \mathbb{M}_{\Sigma}$, and $t=\sum_{k} \sigma_{k} x_{k}$. The product $t \odot t^{\prime}$ is inductively defined by

$$
\begin{aligned}
0 \odot t & =t \\
t \odot t^{\prime} & =\sum_{k} \sigma_{k} x_{k} \odot t^{\prime}=\sum_{k} \sigma_{k}\left(x_{k} \odot t^{\prime}\right) \oplus t^{\prime}
\end{aligned}
$$

We prove the following

Theorem 5.1 The product $\odot$ is associative; thus, $\left\langle\mathbb{M}_{\Sigma}, \odot, 0, f\right\rangle$ is a $\Sigma$-motor and the $\operatorname{map} \lambda=s \circ \nu$ in $\left\langle\mathbb{N}^{\prime}, *, 1, s\right\rangle$ (i.e., $t \mapsto \nu(t)+1$ ) is a morphism in $\Sigma$-Mot. 
Proof. In order to prove associativity, consider three trees $t_{1}, t_{2}, t_{3} \in \mathbb{M}_{\Sigma}$ such that

$$
t_{1}=\sum_{j} \alpha_{j} x_{j}, \quad t_{2}=\sum_{k} \beta_{k} y_{k}
$$

Then, we have

$$
\begin{aligned}
\left(t_{1} \odot t_{2}\right) \odot t_{3} & =\left(\sum_{j} \alpha_{j}\left(x_{j} \odot t_{2}\right) \oplus t_{2}\right) \odot t_{3} \\
& =\left(\sum_{j} \alpha_{j}\left(x_{j} \odot t_{2}\right) \oplus \sum_{k} \beta_{k} y_{k}\right) \odot t_{3} \\
& =\sum_{j} \alpha_{j}\left(\left(x_{j} \odot t_{2}\right) \odot t_{3}\right) \oplus \sum_{k} \beta_{k}\left(x_{k} \odot t_{3}\right) \oplus t_{3} \\
& =\sum_{j} \alpha_{j}\left(x_{j} \odot\left(t_{2} \odot t_{3}\right)\right) \oplus\left(t_{2} \odot t_{3}\right) \\
& =t_{1} \odot\left(t_{2} \odot t_{3}\right)
\end{aligned}
$$

This proves that $\left\langle\mathbb{M}_{\Sigma}, \odot, 0\right\rangle$ is a monoid. Now, consider the map $\lambda$ : trivially, $\lambda(0)=1$. And, given two trees $t_{1}, t_{2}$, we have

$$
\begin{aligned}
\lambda\left(t_{1} \odot t_{2}\right) & =\nu\left(\sum_{j} \alpha_{j}\left(x_{j} \odot t_{2}\right) \oplus t_{2}\right)+1 \\
& =\sum_{j} \nu\left(\alpha_{j}\left(x_{j} \odot t_{2}\right)\right)+\nu\left(t_{2}\right)+1 \\
& =\sum_{j} s\left(\nu\left(x_{j} \odot t_{2}\right)\right)+s\left(\nu\left(t_{2}\right)\right) \\
& =\sum_{j} \lambda\left(x_{j} \odot t_{2}\right)+\lambda\left(t_{2}\right) \\
& =\sum_{j} \lambda\left(x_{j}\right) \lambda\left(t_{2}\right)+\lambda\left(t_{2}\right) \\
& =\left(\sum_{j} \lambda\left(x_{j}\right)+1\right) \lambda\left(t_{2}\right) \\
& =s\left(\sum_{j} s\left(\nu\left(x_{j}\right)\right)\right) \lambda\left(t_{2}\right) \\
& =s\left(\sum_{j} \nu\left(\alpha\left(x_{j}\right)\right)\right) \lambda\left(t_{2}\right) \\
& =s\left(\nu\left(\sum_{j} \alpha_{j} x_{j}\right)\right) \lambda\left(t_{2}\right) \\
& =\lambda\left(t_{1}\right) \lambda\left(t_{2}\right)
\end{aligned}
$$

Besides,

$$
\begin{aligned}
\lambda(\sigma t) & =s(\nu(\sigma t)) \\
& =s(s(\nu(t))) \\
& =s(\lambda(t))
\end{aligned}
$$

This completes the proof.

The map $\lambda$ is just the node-counting map, which we didn't mention before because we're mainly interested in arcs. But arcs are not multiplied by $\odot$. 


\subsection{Tree leaf product}

Another operation on trees, very common in theory of processes, is to attach a copy of a tree to each leaf of another one, as in the following picture

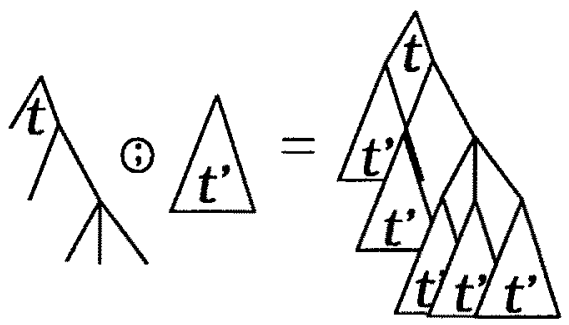

Definition 5.2 Let $t, t^{\prime} \in \mathbb{M}_{\Sigma}$, and $t=\sum_{k} \sigma_{k} x_{k}$. The leaf product $t$ (3) $t^{\prime}$ is inductively defined by

$$
\begin{aligned}
0(3) t & =t \\
t\left(5 t^{\prime}\right. & =\sum_{k} \sigma_{k} x_{k}(3) t^{\prime}=\sum_{j} \sigma_{j}\left(x_{j}(1) t^{\prime}\right)
\end{aligned}
$$

The tree 0 has just one leaf-namely, the root-so it's utterly intuitive that 0 (3) $t$ yields $t$. The leaf product enjoys all the nice properties of the product $\odot$ :

Theorem 5.2 The product (5) is associative; thus, $\left\langle\mathbb{M}_{\Sigma},(3,0, f\rangle\right.$ is a $\Sigma$-motor and the map $\partial_{0}^{\prime}=\partial_{0} \vee \cdot 1$ in $\left\langle\mathbb{N}^{\prime}, *, 1, \mathbb{1}_{\mathbb{N}^{\prime}}\right\rangle$ (i.e., $t \mapsto \partial_{0}(t) \vee 1$ ) is a morphism in $\Sigma$-Mot.

With the same notation of the previous theorem, we have

$$
\begin{aligned}
\left(t_{1}(3) t_{2}\right)(3) t_{3} & =\left(\sum_{j} \alpha_{j}\left(x_{j}\left(3 t_{2}\right)\right)(3) t_{3}\right. \\
& =\sum_{j} \alpha_{j}\left(\left(x_{j} \text { (3) } t_{2}\right)(3) t_{3}\right) \\
& =\sum_{j} \alpha_{j}\left(x_{j}(3)\left(t_{2}(3) t_{3}\right)\right) \\
& =t_{1} \text { (3) }\left(t_{2}(3) t_{3}\right)
\end{aligned}
$$

As for the map $\partial_{0}^{\prime}$, trivially $\partial_{0}^{\prime}(0)=1$. Now, take two trees $t_{1}, t_{2}$, and suppose $\partial_{0}\left(t_{1}\right)>0$, for if $\partial_{0}\left(t_{1}\right)=0$, then $t_{1}=0$ and we would have

$$
\partial_{0}^{\prime}\left(t_{1}(9) t_{2}\right)=\partial_{0}^{\prime}\left(t_{2}\right)=\partial_{0}^{\prime}\left(t_{1}\right) \partial_{0}^{\prime}\left(t_{2}\right)
$$

Otherwise,

$$
\begin{aligned}
\partial_{0}^{\prime}\left(t_{1}\left(j t_{2}\right)\right. & =\partial_{0}\left(\sum_{j} \alpha_{j}\left(x_{j}\left(5 t_{2}\right)\right) \vee 1\right. \\
& =\sum_{j} \partial_{0}\left(\alpha_{j}\left(x_{j}\left(5 t_{2}\right)\right) \vee 1\right. \\
& =\sum_{j}\left(\partial_{0}\left(x_{j}\left(t_{2}\right) \vee 1\right) \vee 1\right.
\end{aligned}
$$




$$
\begin{aligned}
& =\sum_{j} \partial_{0}^{\prime}\left(x_{j}\left(t_{2}\right) \vee 1\right. \\
& =\sum_{j} \partial_{0}^{\prime}\left(x_{j}\right) \partial_{0}^{\prime}\left(t_{2}\right) \vee 1 \\
& =\left(\sum_{j} \partial_{0}^{\prime}\left(x_{j}\right)\right) \partial_{0}^{\prime}\left(t_{2}\right) \vee 1 \\
& =\partial_{0}\left(t_{1}\right) \partial_{0}^{\prime}\left(t_{2}\right) \vee 1 \\
& =\partial_{0}^{\prime}\left(t_{1}\right) \partial_{0}^{\prime}\left(t_{2}\right)
\end{aligned}
$$

The last step is justified since both $\partial_{0}\left(t_{1}\right)$ and $\partial_{0}^{\prime}\left(t_{2}\right)$ are greater than 0 . Besides,

$$
\begin{aligned}
\partial_{0}^{\prime}(\sigma t) & =\partial_{0}(\sigma t) \vee 1 \\
& =\left(\partial_{0}(t) \vee 1\right) \vee 1 \\
& =\partial_{0}^{\prime}(t)
\end{aligned}
$$

This completes the proof.

The map $\partial_{0}^{\prime}$ is a modified leaf-counting map that counts the root as a leaf, so that $\partial_{0}^{\prime}(0)=1$.

\subsection{Tree bottom-right leaf product}

An operation which we will need in order to build a correspondence with binary trees is to attach a copy of a tree to the bottom-right leaf of another tree.

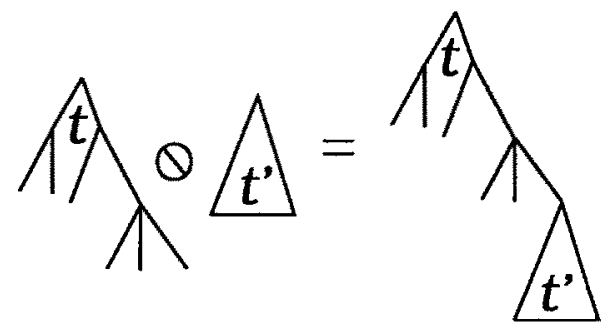

Definition 5.3 Let $t, t^{\prime} \in \mathbb{M}_{\Sigma}$; and $t=\sum_{k} \sigma_{k} x_{k}$. The bottom-right leaf product $t \otimes t^{\prime}$ is inductively defined by

$$
\begin{aligned}
0 \otimes t & =t \\
t \ominus t^{\prime} & =\sigma_{1}\left(x_{1}\right) \oplus \sigma_{2}\left(x_{2}\right) \oplus \cdots \oplus \sigma_{n}\left(x_{n} \oplus t^{\prime}\right)
\end{aligned}
$$

Theorem 5.3 The product $\ominus$ is associative; thus, $\left\langle\mathbb{M}_{\Sigma}, Q, 0, f\right\rangle$ is a $\Sigma$-motor and the map $\nu$ in $\langle\mathbb{N},+, 0, s\rangle$ is a morphism in $\Sigma$-Mot.

The proof of associativity is analogous to the previous theorem. Moreover, $\nu(0)=0$, $\nu(\sigma t)=s(\nu(t))$ and

$$
\nu\left(t_{1} \ominus t_{2}\right)=\nu\left(\alpha_{1} x_{1} \oplus \alpha_{2} x_{2} \oplus \cdots \oplus \alpha_{n}\left(x_{n} \odot t_{2}\right)\right)
$$




$$
\begin{aligned}
& =\nu\left(\alpha_{1} x_{1}\right)+\nu\left(\alpha_{2} x_{2}\right)+\cdots+\nu\left(\alpha_{n}\left(x_{n} \otimes t_{2}\right)\right) \\
& =\nu\left(\alpha_{1} x_{1}\right)+\nu\left(\alpha_{2} x_{2}\right)+\cdots+s\left(\nu\left(x_{n} \otimes t_{2}\right)\right) \\
& =\nu\left(\alpha_{1} x_{1}\right)+\nu\left(\alpha_{2} x_{2}\right)+\cdots+s\left(\nu\left(x_{n}\right)+\nu\left(t_{2}\right)\right) \\
& =\nu\left(\alpha_{1} x_{1}\right)+\nu\left(\alpha_{2} x_{2}\right)+\cdots+s\left(\nu\left(x_{n}\right)\right)+\nu\left(t_{2}\right) \\
& =\nu\left(\alpha_{1} x_{1}\right)+\nu\left(\alpha_{2} x_{2}\right)+\cdots+\nu\left(\alpha_{n} x_{n}\right)+\nu\left(t_{2}\right) \\
& =\nu\left(t_{1}\right)+\nu\left(t_{2}\right)
\end{aligned}
$$

Of course, for the initial abelian $\Sigma$-motor this product does not make any sense.

\section{Binary trees}

In this section we provide an algebraic description of the relationship between trees and binary trees. Binary trees are strictly related to trees, but their recursive definition is slightly different, since it states that there is an empty binary tree, and that every binary tree is a labelled node with a left and a right subtree (see [Knu73]). In order to manage binary trees, we define the following subset of $\mathrm{M}_{\Sigma}$ :

Definition 6.1 The subset $\mathbb{B}_{\Sigma} \subseteq U\left(\mathbb{M}_{\Sigma}\right)$ of binary trees is inductively defined by

$$
\begin{aligned}
0 & \in \mathbb{B} \mathbb{T}_{\Sigma} \\
b, b^{\prime} \in \mathbb{B T}_{\Sigma} & \Rightarrow \sigma b \oplus \sigma b^{\prime} \in \mathbb{B}^{T} \mathbb{T}_{\Sigma}
\end{aligned}
$$

Because of the unique decomposition theorem, from each $b \in \mathbb{B T}_{\Sigma}$ we can recover the associated label and the two subtrees, thus satisfying the recursive definition ${ }^{2}$. Note that we can think of elements of $\mathbb{B P T}_{\Sigma}$ as binary trees labelled on their nodes because we use the same label $\sigma$ for the two arcs under every node. In a sense, we shift up the labels, and in this process the element 0 becomes the empty binary tree.

It is easy in our setting to show that leaf products and bottom-right products of binary trees are still binary trees.

Proposition 6.1 If $b, b^{\prime} \in \mathbb{B T}_{\Sigma}$ then $b(9) b^{\prime}, b \otimes b^{\prime} \in \mathbb{B}^{\prime} \mathbb{T}_{\Sigma}$.

$$
\begin{aligned}
b \oplus b^{\prime} & =\left(\sigma b_{1} \oplus \sigma b_{2}\right) \circledast b^{\prime} \\
& =\sigma\left(b _ { 1 } ( t ^ { \prime } ) \oplus \sigma \left(b_{2}\left(t^{\prime}\right) \in \mathbb{B} \mathbb{T}_{\Sigma}\right.\right. \\
b \otimes b^{\prime} & =\left(\sigma b_{1} \oplus \sigma b_{2}\right) \ominus b^{\prime} \\
& =\sigma b_{1} \oplus \sigma\left(b_{2} \oplus b^{\prime}\right) \in \mathbb{B} \mathbb{T}_{\Sigma}
\end{aligned}
$$

It is well known that every forest (labelled on nodes) can be uniquely represented as a binary tree (labelled on nodes, too) [Knu73]. This is just saying that trees labelled on arcs can be represented as binary trees labelled on nodes. This correspondence is called by Knuth the natural correspondence (not in a categorical sense) between forests and binary trees. The interesting thing is that we can describe this correspondence through a universal map:

\footnotetext{
${ }^{2}$ There is no need in principle to define binary trees in $\mathrm{M}_{\Sigma}$, for instance the initial algebra with a nullary and $|\Sigma|$ binary operators would have done the same job: we choose $B_{\Sigma}$ for a matter of convenience.
} 
Theorem 6.1 The unique map $\mathbb{M}_{\Sigma} \stackrel{c}{\longrightarrow}\left\langle\mathbb{B}^{\prime} \mathbb{T}_{\Sigma}, \otimes, 0, f^{\prime}\right\rangle$, where $f^{\prime}(\sigma,-)=\sigma(-) \oplus \sigma$, is an isomorphism in $\Sigma$-Mot.

To build an inverse, define $d$ as

$$
\begin{aligned}
d(0) & =0 \\
d\left(\sigma b_{1} \oplus \sigma b_{2}\right) & =\sigma d\left(b_{1}\right) \oplus d\left(b_{2}\right)
\end{aligned}
$$

Since

$$
\begin{aligned}
d\left(f^{\prime}(\sigma, b)\right) & =d(\sigma b \oplus \sigma) \\
& =\sigma d(b) \oplus 0 \\
& =\sigma d(b)
\end{aligned}
$$

and

$$
\begin{aligned}
d\left(b \otimes b^{\prime}\right) & =d\left(\sigma b_{1} \oplus \sigma\left(b_{2} \oplus b^{\prime}\right)\right) \\
& =\sigma d\left(b_{1}\right) \oplus d\left(b_{2} \otimes b^{\prime}\right) \\
& =d\left(f^{\prime}\left(\sigma, b_{1}\right)\right) \oplus d\left(b_{2} \oplus b^{\prime}\right) \\
& =d\left(f^{\prime}\left(\sigma, b_{1}\right)\right) \oplus d\left(b_{2}\right) \oplus d\left(b^{\prime}\right) \\
& =d\left(\sigma b_{1} \oplus \sigma\right) \oplus d\left(b_{2}\right) \oplus d\left(b^{\prime}\right) \\
& =d\left(\left(\sigma b_{1} \oplus \sigma\right) \otimes b_{2}\right) \oplus d\left(b^{\prime}\right) \\
& =d\left(\sigma b_{1} \oplus \sigma b_{2}\right) \oplus d\left(b^{\prime}\right) \\
& =d(b) \oplus d\left(b^{\prime}\right)
\end{aligned}
$$

we have $d \circ c=\mathbf{1}_{\mathbb{M}_{\Sigma}}$ by initiality. But now

$$
\begin{aligned}
(c \circ d)(b) & =(c \circ d)\left(\sigma b_{1} \oplus \sigma b_{2}\right) \\
& =c\left(\sigma d\left(b_{1}\right) \oplus d\left(b_{2}\right)\right) \\
& =f^{\prime}\left(\sigma, c\left(d\left(b_{1}\right)\right)\right) \otimes c\left(d\left(b_{2}\right)\right) \\
& =f^{\prime}\left(\sigma, b_{1}\right) \otimes b_{2} \\
& =\left(\sigma b_{1} \oplus \sigma\right) \otimes b_{2} \\
& =\sigma b_{1} \oplus \sigma\left(0 \otimes b_{2}\right) \\
& =b
\end{aligned}
$$

Notice that Bénabou had already the unlabelled version of this theorem.

This "natural correspondence" admits a sort of geometrical description (see [Knu73]), but the universal map given here is all you need: it describes the entire process in just one gulp. Here's an example: 


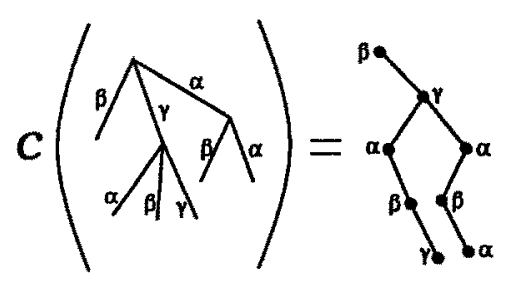

\section{Conclusions}

We would like to make some final comments on the content of the present paper and to sketch briefly further developments of the subject.

After having introduced our labelled version of Bénabou's notion of motor, we showed with several examples of counting maps, both qualitative and quantitative, the flexibility of this algebraic structure. Maps which should have been defined through a recursive definition involving several steps can now be described in just one shot, simply assigning a correct motor structure to the codomain. Besides, being able to define such a structure requires no calculations at all, but just a little bit of thought and a clear understanding of what is wanted. In a sense, using maps out of the initial motor forces a precise analysis of the algebraic property that one wishes to investigate.

From a purely mathematical point of view, Bénabou's approach provides a presentation-free, invariant description of finite trees, dispensing us with clumsy manipulations of strings, terms, graphs or whetever else. And then again, the powerful inductive structure allows for easy and, above all, natural proofs which can be carried on using only preservation of the identity, of the product and of the action. Further, as the reader probably noticed, our calculus is after all just a rigorous formalization of the common usages in this kind of computations.

Then we investigated some of the possible "external" algebraic characterizations of $\mathbb{M}_{\Sigma}$. Besides the obvious mathematical interest they have in their own right, they provide some tools to manage directly and elegantly the applications. Let us also point out that the colimit construction of section 3.2 has a meaning which is maybe deeper than it seems: in fact, the dualization of every step of the construction leads to a new object, which will be the tool for studying infinite trees and will be extensively investigated in a subsequent paper.

We described (unary and binary) operations on trees which have obviously a wide range of applications in CCS-like approaches to concurrency. For instance, in [DKV] we showed how to build canonical representatives for Milner's observational equivalence using a map out of $\mathbb{M}_{\Sigma}$.

Let us finally remark that the very nature of the algebraic structure we use does not allow for a treatement of continuous trees (see $[\mathrm{KL}]$ ), but we hope we convinced the reader about the usefulness and the beauty of the $\Sigma$-motors approach in a large variety of applications related to trees. 


\section{References}

[Bén90] J. Bénabou. Lectures held at the Mathematics and Computer Science Departments of the University of Milano, and talk at the Category Theory ' 90 Conference in Como. 1990.

[BK85] J.A. Bergstra and J.W. Klop. Algebra of communicating processes with abstraction. Theoretical Computer Science, 37(1):77-121, 1985.

[DKV] P. Degano, S. Kasangian, and S. Vigna. Applications of the calculus of trees to process description languages. Submitted.

[KL] S. Kasangian and A. Labella. Continuous time agents. To appear in Proceedings MFPS '91.

[KL90] S. Kasangian and A. Labella. Enriched categorical semantics for distributed calculi. Journal of Pure and Applied Algebra, 1990. To appear.

[Knu73] D.E. Knuth. The Art of Computer Programming. Addison-Wesley, 1973.

[KV] S. Kansangian and S. Vigna. Trees in a distributive category. To appear in Proceedings CT '90.

[MB67] S. Mac Lane and G. Birkhoff. Algebra. Macmillan, 1967.

[Mil80] R. Milner. A Calculus of Communicating Systems. Number 92 in Lecture Notes in Computer Science. Springer-Verlag, 1980.

[Win84] G. Winskel. Synchronization trees. Theoretical Computer Science, 34:33-82, 1984. 